\title{
MOdULATION OF CUTANEOUS RESPONSES IN THE CUNEATE NUCLEUS OF MACAQUES DURING ACTIVE MOVEMENT
}

Qinpu He ${ }^{1}$, Christopher S. Versteeg ${ }^{23}$, Aneesha K. Suresh ${ }^{1}$, Joshua Rosenow ${ }^{6}$, Lee E. Miller ${ }^{345}$, and Sliman J. Bensmaia ${ }^{1,7,8 *}$

${ }^{1}$ Committee on Computational Neuroscience, University of Chicago, Chicago, IL

${ }^{2}$ Department of Neuroscience, Northwestern University, Chicago, IL

${ }^{3}$ Department of Biomedical Engineering, Northwestern University, Evanston, IL

${ }^{4}$ Department of Physical Medicine and Rehabilitation, Northwestern University, Chicago, IL

${ }^{5}$ Shirley Ryan AbilityLab, Chicago, IL

${ }^{6}$ Department of Neurological Surgery and Neurology, Northwestern University, Chicago, IL

${ }^{7}$ Department of Organismal Biology and Anatomy, Chicago, IL

${ }^{8}$ Grossman Institute for Neuroscience, Chicago, IL

¥sliman@uchicago.edu

\section{ACKNOWLEDGMENTS}

This work was supported by NINDS grants NS095162 and NS122333. 


\begin{abstract}
To achieve stable and precise movement execution, the sensorimotor system integrates exafferent sensory signals originating from interactions with the external world and reafferent signals caused by our own movements. This barrage of sensory information is regulated such that behaviorally relevant signals are boosted at the expense of irrelevant ones. For example, sensitivity to touch is reduced during movement - when cutaneous signals caused by skin stretch are expected and uninteresting - a phenomenon reflected in a decreased cutaneous responsiveness in thalamus and cortex. Some evidence suggests that movement gating of touch may originate from the cuneate nucleus (CN), the first recipient of signals from tactile nerve fibers along the dorsal column-medial lemniscus pathway. To test this possibility, we intermittently delivered mechanical pulses to the receptive fields (RFs) of identified cutaneous CN neurons as monkeys performed a reach-to-grasp task. As predicted, we found that the cutaneous responses of individual $\mathrm{CN}$ neurons were reduced during movement. In contrast, cutaneous signals from neurons with RFs on the hand were enhanced during reach. We conclude that tactile signals are already modulated in the first processing stage along the somatosensory neuraxis. Furthermore, the sign and magnitude of this modulation depends on the behavioral relevance of the sensory signals.
\end{abstract}

\title{
INTRODUCTION
}

Touch is typically engaged during goal-directed interactions with objects and conveys information about the objects and about our interactions with them (Goodman \& Bensmaia, 2018; Johansson \& Flanagan, 2009). However, touch signals are also triggered by the movement itself (Aimonetti et al., 2007, 2012; Edin \& Abbs, 1991; Johansson, 1978). For example, as we reach for an object, nerve fibers that innervate the skin of the shoulder and elbow become activated by the skin stretch that accompanies the joint deflections (Costanzo \& Gardner, 1981). To the extent that the cutaneous signals associated with movement convey information about limb posture, these signals are largely redundant with those from the muscles and tendons. Accordingly, it may make sense to suppress cutaneous signals triggered by movement-related skin stretch so that these do not interfere with signals arising from object interactions.

Consistent with this hypothesis, sensitivity to cutaneous stimulation has been shown to be reduced during reaching (Chapman et al., 1987). The neural correlates of this sensory gating have been observed in local field potentials (or the equivalent) measured in the spinal cord (Confais et al., 2017; Seki \& Fetz, 2012), medial lemniscus, thalamus (Chapman et al., 1988), and somatosensory cortex of macaques (Seki \& Fetz, 2012) and humans (Kurz et al., 2018). The cuneate nucleus (CN) is the first stage of processing of tactile signals along the dorsal column medial lemniscus pathway and receives abundant descending inputs from cortex (Conner et al., 2021; Loutit et al., 2020). Accordingly, this tiny structure in the brainstem is well positioned to play a role in task-dependent gain modulation. However, this suppression has not previously been characterized at the single-cell level so it is unclear (1) whether all CN neurons are subject to gating or only some are; (2) whether gating is deployed homogeneously over the entire body map or rather sculpted depending on the body location of incoming signals; and (3) whether this gating is all-or-none or graded according to movement parameters. 
To address these questions, we had rhesus macaques perform a reach-tograsp task while we delivered tactile stimuli intermittently to the identified locations of the receptive fields (RFs) of cutaneous neurons in $\mathrm{CN}$. As the animals performed the task, we measured neuronal responses, tracked limb movements, and measured the forces exerted by the monkey on the manipulandum. We found that cutaneous responses in $\mathrm{CN}$ were systematically suppressed during movement for neurons with RFs on the arm but were elevated for neurons with RFs on the palmar surface of the hand, where object contact was anticipated. In some cases, cutaneous responses of arm CN neurons were also suppressed during maintained grasp force. Finally, gating was observed for some units even before movement initiation Sand was not observed when the arm was moved passively, demonstrating the topdown origins of the gating. We conclude that $\mathrm{CN}$ plays a major role in the movement gating of tactile signals.

\section{RESULTS}

We recorded the responses of $29 \mathrm{CN}$ cutaneous neurons, those which responded to light touch over a restricted patch of skin, from a total of five Rhesus Macaques. Of these, 18 neurons had RFs on the arm (including one on the dorsal surface of the hand) and 11 on the palmar surface of hand.

Animals were trained to perform a reach-and-grasp task. On each trial, an LED cued the animal to reach to and grasp a joystick-like manipulandum and apply a force the magnitude of which was also cued by the multi-LED display. The animal obtained a water reward if it held the specified force for the required duration (see Methods for details). After obtaining the reward, the animal returned its arm to the arm rest and the next trial was initiated. Each trial was split into different epochs: rest, cue, reach, and grasp. To assess whether $\mathrm{CN}$ responses are state dependent, we compared stimulus-evoked responses during rest to those evoked during the other three epochs (Figure $1 \mathrm{~A}$ ). To this end, we first aligned the response
A

B

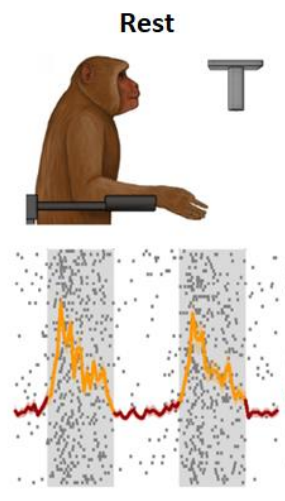

am unit 1 Rest

究

Figure 1. Behavioral task and neural data. A| Behavioral task. The monkey placed its free arm onto the arm rest for an interval of variable duration (rest epoch before it initiated a trial cued by an LED. The animal then reached tovard (reach epoch) and grasped the manipulandum, applied the cued force (force epoch), received a water reward, then returned its arm to the arm rest to wait for the start of the next trial. Tactors placed over identified receptive fields vibrated every $200 \mathrm{~ms}$ for $100 \mathrm{~ms}$ throughout the entire task. Responses of one exemplar arm tactile $\mathrm{CN}$ neuron over 50 trials depicted below the behavioral diagram. The peri-stimulus time histograms are overlaid with orange traces denoting the stimulation-induced mean response and red traces the corresponding baseline activity in between vibratory stimuli. B| Demeaned average responses of two cutaneous CN neurons with RFs on the arm. Gray shading denotes the stimulation epoch. Orange, purple, green, and blue lines stand for the average responses during rest, cue, reach, and grasp epochs, respectively. The cue epoch is that between the onset of the LED and the start of the reach. C| Same as B but for a palm neuron. Tactile responses during grasp are not shown, as the epoch. vibration-elicited responses are contaminated by the grasp during this 
A

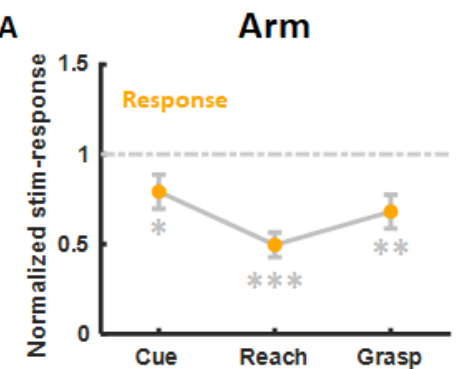

C

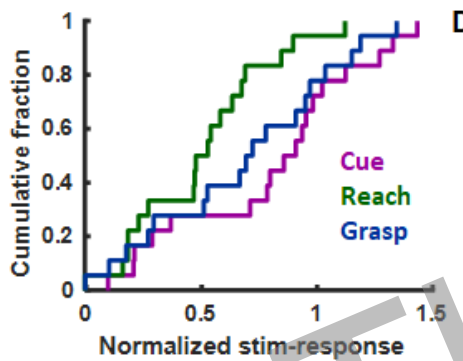

E

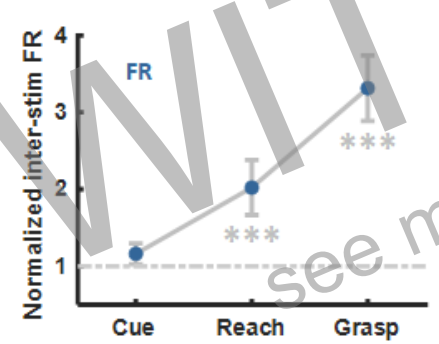

B

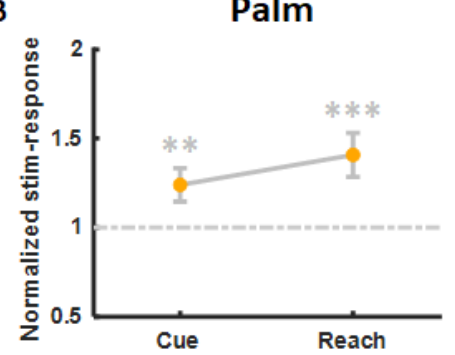

D
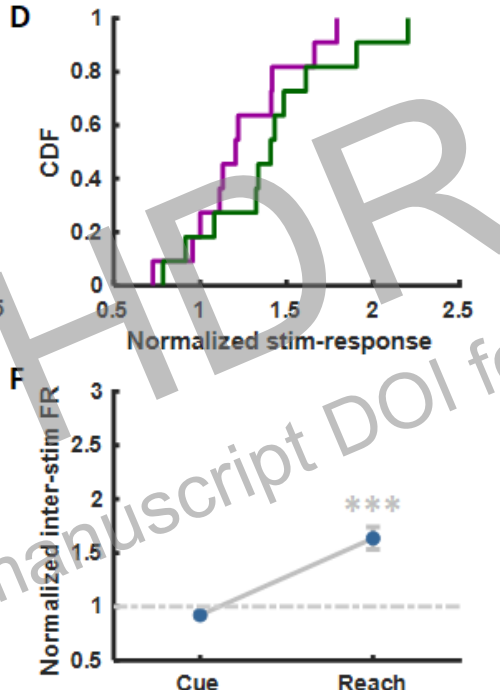

Figure 2. Stimulation-evoked and baseline responses of arm and palm cutaneous neurons. A| Mean normalized stimulation-evoked responses of all cutaneous neurons with RFs on the arm $(n=18)$ to stimulation during the cue, reach, and grasp epochs normalized to the rest responses. Responses were significantly reduced during all three epochs. $(* \mathrm{p}<0.05, * * \mathrm{p}<0.01, * * * \mathrm{p}<0.001$, significant deviation from rest epoch, Wilcoxon signed-rank test). B| Mean stimulation-evoked responses of all cutaneous neurons with RFs on the palmar surface of the hand $(n=11)$ during different epochs. Responses were significantly enhanced during the cue and reach epochs. C| Cumulative histograms of normalized stim-responses of all arm neurons during the three behavioral epochs. A total of 5, 12, and 7 out of 18 total arm cutaneous units showed significantly suppression during cue, reach, and grasp period, respectively ( $\mathrm{p}<0.05$, Wilcoxon rank sum test). D| Same as panel C but for all palm neurons. A total of 4 and 5 out of 11 palmar cutaneous units exhibited significantly elevated responses during the cue and reach periods, respectively. E| Mean baseline response to the same arm neurons as in A. Baseline firing rates were significantly elevated during reach and grasp compared to rest. F| Mean baseline responses of palm units.

of each neuron to the onset of each stimulus, computed the evoked firing rate (bottom row of Figure $1 \mathrm{~A}$ ), then averaged these separately for each epoch (Figure 1B,C). We also computed the mean firing rate during the inter-pulse intervals to assess how the baseline response (without the tactor) varied across epochs.

Behaviorally dependent modulation of cutaneous responses

tollgauge the response modulation in each epoch, we divided the mean stimulusevoked response by the mean stimulus-evoked response during rest. Accordingly, values greater than 1 indicated elevated responses with respect to rest whereas values less than 1 indicated suppression. To the extent that $\mathrm{CN}$ responses exhibit movement gating, the stimulation-evoked response of cutaneous neurons would be weaker during the reach epoch and perhaps during the grasp epoch compared to their rest counterparts. As predicted, the tactile responses of arm cutaneous units were significantly weaker during reach and grasp (Figure $2 A, B)$, as evidenced by normalized stim-responses

that fall below 1 . The attenuation of the responses occurred even before the start of movement, as shown by the decreased responses during the cue epoch. Note that the baseline responses during the cue and rest epochs were equivalent, confirming that the monkey was not moving during the cue epoch. The presence of such gating during preparation phase implicates descending inputs. We found that the responses of $77 \%(14 / 18)$ of the cutaneous units with RFs on the arm were significantly suppressed during 
at least one behavioral epoch: $11 \%$ throughout all three, 33\% throughout two, and another 33\% during only one (Figure $2 \mathrm{C}$ ).

Note, however, that the baseline responses - those measured during the inter-stimulus intervals - were elevated during the reach and grasp epochs (Figure 2E), opening up the possibility that the reduced activity during the movement and force epochs reflected saturation of the responses when reafferent and exafferent signals co-occur. However, there was no relationship between the strength of the movement gating and the magnitude of baseline (non-stimulus evoked) activity (Supplementary Figure 2). That is, neither those neurons whose baseline response increased the most during movement nor those whose baseline firing rate was highest systematically exhibited the strongest suppression of the stimulus-evoked during movement. Hence, movement induced gating is not a trivial consequence of response saturation.

\section{Dependence of modulation on behavioral variables}

The suppression of cutaneous sensitivity at the lemniscal, thalamic, and cortical levels has been shown to depend on the speed of movement (Chapman et al., 1988; Ghez \& Pisa, 1972). With this in mind, we examined the degree to which the suppression was modulated by behavioralparameters, including movement speed as well as applied force. We found that, of the 18 cutaneous neurons with RFs on the arm, six were modulated significantly more strongly at higher speeds during the reach epoch (Supplementary Figure 1A). The influence of grasp force on modulation for proximal limb neurons (during the grasp epoch) also varied: The responses of four neurons were significantly elevated while those of two neurons decreased significantly at higher grasp forces (Supplementary Figure 1B). Thus, the dependence of the gain modulation on behavioral variables is heterogeneous: The modulation is all-ornone for some neurons and graded with behavior for others.

Modulation of cutaneous neurons with RFs on the hand

All the analyses described above were performed on cutaneous neurons with RFs on the arm. A subset of recorded neurons had cutaneous RFs on the glabrous skin (palmar surface) of the hand. Unlike their counterparts with receptive fields on the arm, CN neurons with RFs on the glabrous skin exhibited systematically elevated stimulusevoked responses during the reach epoch (Figure 2 B\&D). Responses of hand neurons during grasping epochs were not analyzed because contact with the manipulandum contaminated the stimulus-evoked responses. In total, the responses of $10 \%$ of palm units were consistently
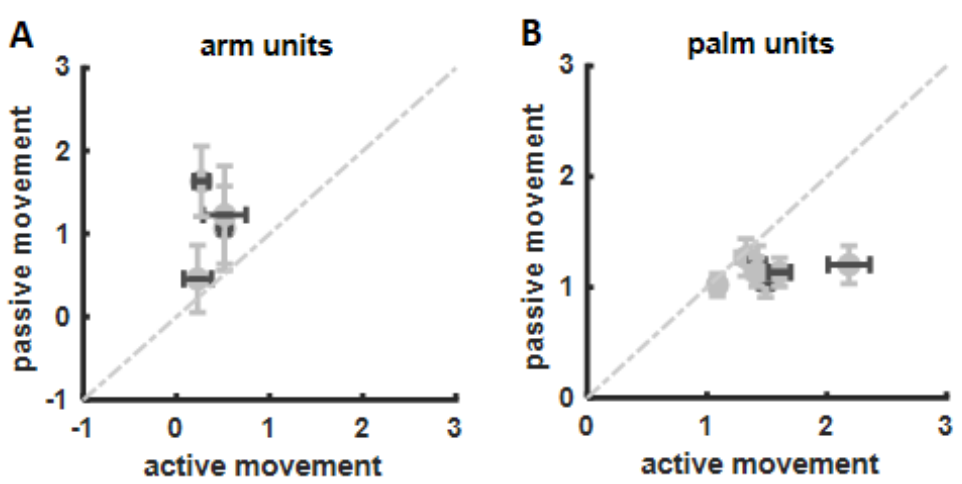

Figure 3. Stimulation-evoked responses of arm and palmar cutaneous neurons under active vs. passive movement. A| Mean stimulation-evoked responses of all cutaneous neurons with RFs on the arm $(n=4)$ to stimulation during three event epochs normalized to the rest responses. A darker color error bar on the abscissa or ordinate denotes a significant movement-related modulation under active or passive conditions, respectively. None of the four units showed significantly reduced responses during passive manipulations. B| Mean stimulation-evoked responses of all cutaneous neurons with RFs on the palm $(n=6)$. None of the six units showed significantly elevated responses during passive manipulations. (Wilcoxon rank sum test). 
elevated throughout all three behavioral epochs, $27 \%$ throughout two epochs, and another $27 \%$ during only one (Figure 2D).

The modulation of cutaneous sensitivity thus seems to depend on RF location, which may reflect the task relevance of signals from different body regions: Signals from the proximal limb are likely reafferent and not particularly informative, whereas signals from the hand carry information about contact with the manipulandum and are thus critical to task performance.

\section{Modulation during imposed movement}

To assess the degree to which the observed task-related modulation of cutaneous sensitivity was mediated by top-down signals, we compared the stimulus-evoked responses during active movements to those during passively imposed movements. To the extent that gating was caused by top-down signals, we expected it to be absent in the passive condition. We found that, indeed, the stimulus-evoked responses of $\mathrm{CN}$ neurons with RFs on the arm during imposed arm movements were not significantly different from those at rest (Figure $3 A ; p=0.96$, Wilcoxon rank sum test) and were significantly elevated compared to those measured during active reach $(p<0.001)$. Similarly, the responses of $C N$ neurons with RFs on the glabrous skin were not significantly modulated during passively imposed movements of the $\operatorname{arm}(p=0.32)$ and were significantly lower than those measured during active reach (Figure $3 B ; p<0.001)$. That movement gating of sensory signals is only observed during actively generated movements is consistent with the hypothesis that this gating originates centrally.

\section{DISCUSSION}

We show that the cutaneous sensitivity of $\mathrm{CN}$ neurons with RFs on the proximal limb is systematically suppressed during reaching movements. In contrast, the sensitivity of $\mathrm{CN}$ neurons with RFs on the hand is either unaffected or elevated. In some neurons (39\%), cutaneous sensitivity is also modulated as the animal applies isometric forces on the manipulandum, in the absence of overt movement. The modulation of $\mathrm{CN}$ responses has a central origin as evidenced by the fact that it is observed in $28 \%$ of arm and $36 \%$ of palm units even before movement - i.e., before reafference is engaged - and only during active reaching movements. The strength of the modulation varies across neurons from 25 to $95 \%$ suppression for the arm and from $20 \%$ to $220 \%$ elevation for the hand. The dependence of modulation on behavioral parameters - including movement speed or exerted force - also varies widely across neurons: In most neurons the modulation is all-or-none whereas, in a small subpopulation, the modulation is graded by the speed or magnitude of exerted force. Our results are consistent with the view that top-down signals sculpt the sensory input to squelch behaviorally irrelevant and possibly distracting cutaneous signals and enhance behaviorally relevant ones.

\section{Sculpting of cutaneous signals according to task relevance}

We show that cutaneous sensitivity is not homogeneously gated over the entire body during a reaching movement. CN neurons with RFs on the arm are suppressed whereas neurons with RFs on the glabrous skin are sensitized. This spatial specificity of gating has also been observed in human psychophysical experiments: tactile thresholds near the moving segment increased most and less so farther away (Post et al., 1994; Williams et al., 1998). The differential modulation of signals from different body regions may reflect their differential utility in accomplishing a task (Juravle et al., 2017). In a reach-to-grasp task, cutaneous signals from the palmar surface of the hand signal contact with the manipulandum and trigger 
the end of the grasp (Johansson \& Flanagan, 2009), whereas signals from the arm reflect reafference and are less behaviorally relevant. The observed modulation would then enhance the task-relevant hand signals and suppress the task-irrelevant arm signals. Consistent with this interpretation, detection thresholds at the forearm have been shown to increase during a reach-to-grasp while those of distal digits did not (Colino et al., 2014). The task-dependence of modulation has been observed in a variety of other contexts, not limited to use of the arm (Juravle et al., 2013; Staines et al., 2000, 2002).

\section{Dependence of modulation on behavioral variables}

We find that the magnitude of the modulation is largely independent of behavioral variables, such as movement speed and applied force. Indeed, only a few CN neurons exhibited cutaneous responses whose strength depended on the speed of the reach or the force applied on the manipulandum. For most neurons, the modulation was all-or-none. The mixture of behaviorally graded and all-or-none modulation at the single-cell level is consistent with the observation that lemniscal potentials measured in cats and primates are more suppressed at higher velocities (Chapman et al., 1988; Ghez \& Pisa, 1972; Rushton et al., 1981). Indeed, field potentials reflect the mixed activity of neurons that exhibit speed-dependent and speed-independent suppression. Similarly, sensory thresholds measured in human observers have been shown to increase with movement speed (Angel \& Malenka, 1982; Schmidt et al., 1990). In contrast, responses reflecting whisker contact in the trigeminal nucleus of rats seem to be suppressed during active whisking in all-or-none fashion, though this phenomenon was not analyzed at the single-cell level, leaving open the possibility that a subpopulation with graded modulation exists (Chakrabarti \& Schwarz, 2018).

Studies of the effect of force exertion on cutaneous sensitivity have yielded mixed results. Varying isometric force had no effect on cutaneous signals measured in the somatosensory cortex of humans (Rushton et al., 1981) or on medial lemniscus potentials in cats (Ghez \& Pisa, 1972). However, forces exerted by the lower limb did modulate cutaneous signals in cortex in a graded fashion (Cohen \& Starr, 1985; Sakamoto et al., 2004; Wasaka et al., 2005). In line with the latter result, psychophysical experiments with human observers revealed an increase in detection thresholds during higher isometric force production (Post et al., 1994). This seeming heterogeneity across studies may reflect the fact that only a small subpopulation of $\mathrm{CN}$ neurons exhibits a force-dependent modulation so these effects may be unpredictable in any small sampling of neurons, depend on the specific ranges of forces applied, and vary depending on which muscles are activated and where the tactile stimulus is applied.

\section{Central origins of modulation}

The modulation of tactile sensitivity we observed likely has a central origin. Indeed, cutaneous responses are suppressed or enhanced before movement onset and this modulation is not observed during passive movements. A putative circuit to mediate this centrally driven modulation has been identified in mice. The $\mathrm{CN}$ can be divided into two regions: a core that receives the bulk of the input from the periphery and an outer shell that comprises mostly inhibitory interneurons (Conner et al., 2021). Each region receives a distinct pattern of projections from cortex: The core receives excitatory inputs from somatosensory cortex whereas the shell receives both excitatory and inhibitory input from a variety of motor structures. Together, these top-down pathways can account for the observed modulation of cutaneous sensitivity. Excitatory projections from motor cortex to inhibitory neurons in the shell region can account for the suppression of cutaneous signals. Excitatory projections from somatosensory cortex to the core region or inhibitory projections from motor cortex to the shell region can mediate the observed elevation of cutaneous sensitivity. While the relevant anatomy of rodents and primates differs, primate $\mathrm{CN}$ also 
receives a mix of excitatory and inhibitory inputs from cortex (Biedenbach, 1972; Loutit et al., 2020). Thus, similar circuitry likely underlies the modulation observed here.

\section{MetHODS}

\section{Experimental apparatus}

A joystick was mounted to a high-sensitivity force sensor (ATI Six-Axis Force/Torque Transducer). Two arrays of LEDs were placed above the sensor, one to cue the level of grasp force the monkey needed to exert to obtain a reward and the other reflecting the actual force applied to the manipulandum. Tactile stimulation was delivered using DC coreless vibration motors ( $7 \mathrm{~mm} \times 25 \mathrm{~mm}$, Speed: $8000-24000$ RPM), which were fixed to various locations on the monkeys' arms, each corresponding to the identified RF of a $\mathrm{CN}$ neuron. The onset of movement was signaled by photoresistors embedded in the arm rest of the monkey chair and arm and hand movements were tracked using a camera and two wireless motion trackers attached to the upper and lower arm (Xsens 3D Motion Tracking System, Enschede, Netherlands).

\section{Behavioral paradigm}

We trained five rhesus macaques (four males, one female, ages 5-10 yr) to perform a reach-and-grasp task (Figure 1A). Monkeys were seated upright at the experimental chair, with one arm restrained and the other free, facing LEDs and the joystick. The animal placed its free arm onto the arm rest for a random period of $0.5-2 \mathrm{~s}$ (rest epoch) to initiate a trial. A trial began when the first row of LEDs lit up, signaling the monkey to reach towards the manipulandum. The LED prescribed the amount of force, which varied across trials, that the monkey needed to apply to the manipulandum on that trial. Application of a load on the joystick activated the second row of LEDs, with the number of activated LEDs proportional to the applied force. If the amount of force exceeded the required force for a required interval (varying between $0.1-2.0 \mathrm{~s}$; hold), the monkey would receive a liquid reward. The animal then returned its arm to the arm rest for $2 \mathrm{~s}$, received a second water reward, the LEDs were turned off, and the animal waited for the initiation of the next trial. Recordings from the first reward onset to the end of every trial were not included in data analysis to minimize the influence of task-unrelated movement, as the animal fidgeted as it consumed its rewards.

Before the start of every recording session, we performed receptive field mapping to locate tactile $\mathrm{CN}$ units. We classified a unit as cutaneous if it responded to gentle stroking of the skin but not to lengthening and palpation of the muscle underneath the skin. During the recording sessions, $60-100 \mathrm{~Hz}$ vibrations were applied to the RFs of identified CN units in regular intervals (100-ms on, 100-ms off). The vibrating tactors were firmly attached to the locations on the arm corresponding to the RFs of the neurons using glue then secured in place with three layers of vet wrap. The arm was shaved at each RF location before the experiments began. As the animal performed the task, arm movements were tracked using two accelerometers attached to the proximal and distal arms with Velcro bands. Data recorded from days when the tactors or accelerometers were found to have moved at the end of recording sessions were excluded. A camera monitored the monkey's behavior throughout the recordings. A publicly available motion-tracking package was used to track the monkey's arm movements and to infer reaching speeds based on these (DeepLabCut, Mathis et al., 2018).

On some trials, we manually manipulated the monkey's arm to mimic reaching movements. Specifically, we followed the same timing as in the active reaching task (Figure 1), except that the experimenter held the monkey's arm to move it toward the manipulandum and return it to the arm-rest. The experimenter avoided contact with the tactors during these passive trials.

Neural Data Acquisition 
We implanted 96-channel iridium-oxide Utah arrays (UEA) (Blackrock Microsystems, Inc., Salt Lake City, UT) in all five monkeys. We used customized $8 \times 12$ shank rectangular array with $1.5 \mathrm{~mm}$ electrode length and 9-cm wire bundle between array and pedestal. Neural data were acquired using a digital CerePlex $\mathrm{E}$ headstage and CerePlex Direct acquisition system (Blackrock Microsystems). We bandpass-filtered neural signals between $250 \mathrm{~Hz}$ and $5000 \mathrm{~Hz}$ and set the voltage threshold for every channel manually before the start of every recording session. We recorded the time of every threshold crossing on every channel and saved the neural waveforms over a 1.6-ms window for off-line spike sorting (Plexon, Dallas, TX).

We simultaneously recorded timestamps that indicated when stimulation and behavioral events occurred, along with the neural data while the monkey performed the task.

\section{Surgical Procedures}

All experimental protocols were reviewed and approved by the University of Chicago Animal Care and Use Committee and complied with the National Institutes of Health Guide for the Care and Use of Laboratory Animals. We anesthetized the monkeys using ketamine $\mathrm{HCl}(3 \mathrm{mg} / \mathrm{kg} \mathrm{im})$ and dexmedetomidine $(75 \mu \mathrm{g} / \mathrm{kg})$ and fixed the animal's head in a stereotaxic frame such that neck was flexed to $\sim 75^{\circ}$ relative to the body to allow for maximum exposure of brainstem. We first made a midline incision from the occipital bone to approximately $\mathrm{C} 1 \sim \mathrm{C} 3$ and removed posterior cervical muscles along the midline using cautery. We secured the pedestal to the skull with bone screws such that the routing of the wire bundle lead between it and the array in the brainstem would be flexible enough to enable normal neck movements. We then exposed the foramen magnum and the occipitocervical dura using cautery and sharp dissection. We identified the Obex and used the stereotaxic frame to target 2-mm lateral to midline and 2-mm anterior to Obex to aim for maximum coverage of distal limb cutaneous regions of upper body (Darian-Smith \& Ciferri, 2006; Loutit et al., 2020; Qi \& Kaas, 2006; Suresh et al., 2017, 2021; Versteeg et al., 2021). The UEA was then implanted into the brainstem using a pneumatic inserter (Blackrock Microsystems, Inc.).

\section{Data Analysis}

\section{Defining the task epochs}

To evaluate the epoch-dependent modulation of responses, we first defined each behavioral epoch by using the output the photoresistor, accelerometer, and force gauge. Reaching onset was defined as the first time when photoresistors on the arm rest were uncovered while reaching offset was defined as a rise of force on the manipulandum above a threshold. The start and end of reach were accompanied by (and confirmed via) measurements of the acceleration of the arm.

After finding the onset and offset of the reach, we defined four movement-related epochs for analysis: (1) rest, from the start of the photoresistors coverage until the onset of the cue; (2) cue, from the onset of the cue to the onset of movement (gleaned from the photoresistor signal); (3) reach, from the onset of movement to the onset of force production on the manipulandum; (4) grasp, from the onset of force production to the water reward trigger (Figure 1A). To minimize the possibility of movement during the rest and cue epochs, we only included stimulation snippets surrounded by baseline FRs that were within three standard deviations of the mean FR during these two epochs.

\section{Gauging the extent of modulation}

First, we computed the firing rate of each neuron during each 100 -ms stimulus period and the firing rate 50-ms before and 50-ms after the stimulus (the baseline). Then, we computed the mean difference between stimulus and baseline periods for each epoch (Figure 1B\&C). We compared the rest responses to those of the other three epochs (cue, reach, and grasp) using the Wilcoxon signed-rank test. Finally, we divided the mean stimulus-evoked response in each epoch by the mean response during rest as an index of the modulation effect (Figure 2). 


\section{Gauging the dependence of modulation on movement speed}

To investigate the influence of movement speed on modulation, we derived movement speed during each reach using DeepLabCut (Mathis et al., 2018). First, we manually labeled fifty randomly sampled video frames from all monkeys to train the network. From these networks, we reconstructed the time-varying arm postures and derived from these the time-varying movement speeds. We split the speeds into quartiles and computed the mean normalized stimulus-evoked responses for each quartile (Supplementary Figure 1). Trials during which movement trajectories were blocked in videos were excluded.

\section{Gauging the dependence of modulation on applied force}

To study the influence of force exertion on modulation, we split the grasp forces into those above and those below the median force. We then averaged the stimulus-evoked responses in each group and compared them (Supplementary Figure 1).

Assessing the role of response saturation

To verify that the apparent suppression of cutaneous responses during movement and force epochs was not simply due to response saturation, we calculated baseline firing rates (at rest and during movement) and examined whether the strength of the effect was related to baseline, computed either during rest or throughout the movement. If the suppression were due to response saturation, we expected that neurons with higher baseline firing rates or neurons whose firing rate increased the most during movement would exhibit the strongest modulation (Supplementary Figure 2). No such relationship was observed. 


\section{SUPPLEMENTARY FIGURES}
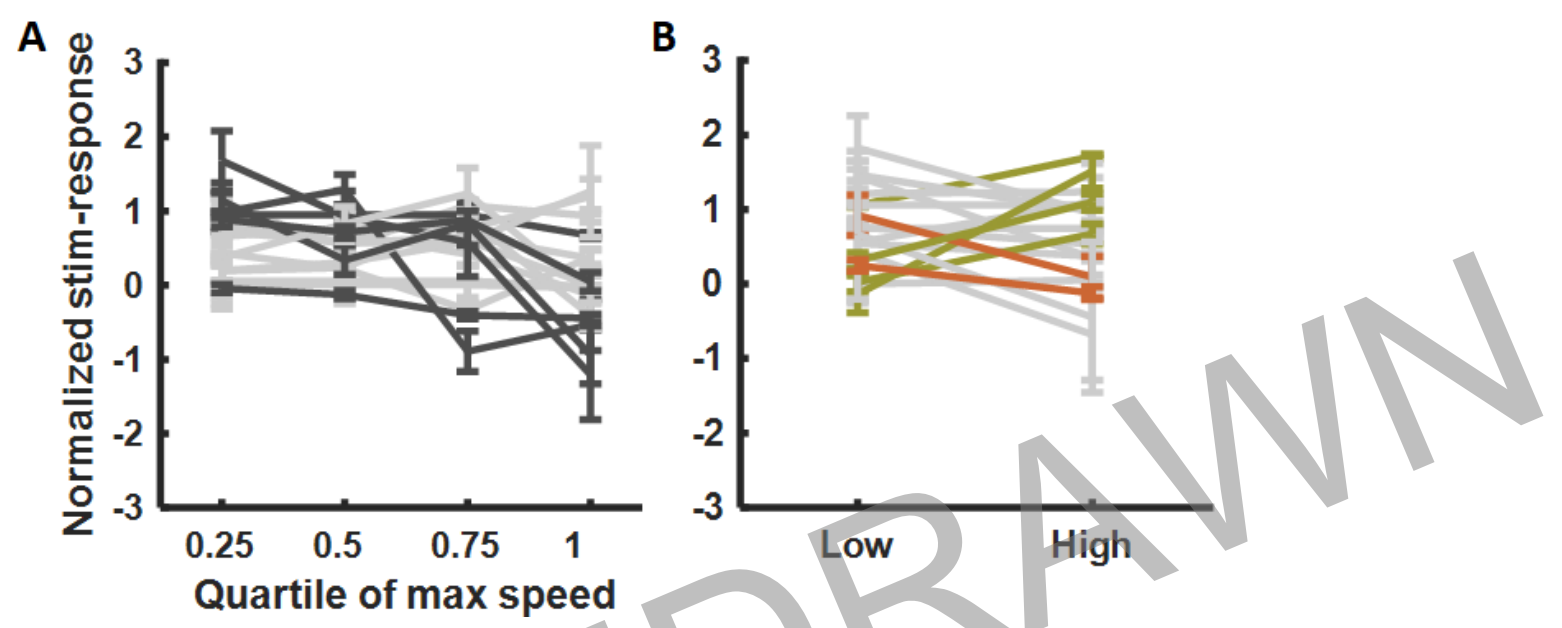

Supplementary Figure 1. Dependence of modulation on movement parameters. A| Dependence of modulation on speed. Each line shows the responses of one cutaneous arm neuron. Dark grey traces denote neurons for which the modulation was significantly dependent on speed. B| Dependence of modulation on force. Green traces denote neurons whose cutaneous sensitivity was significantly elevated on high force trials; red traces denote neurons whose sensitivity was significantly suppressed sensitivity during high-force trials; grey traces denote neurons whose sensitivity was not significantly different across force levels.

A

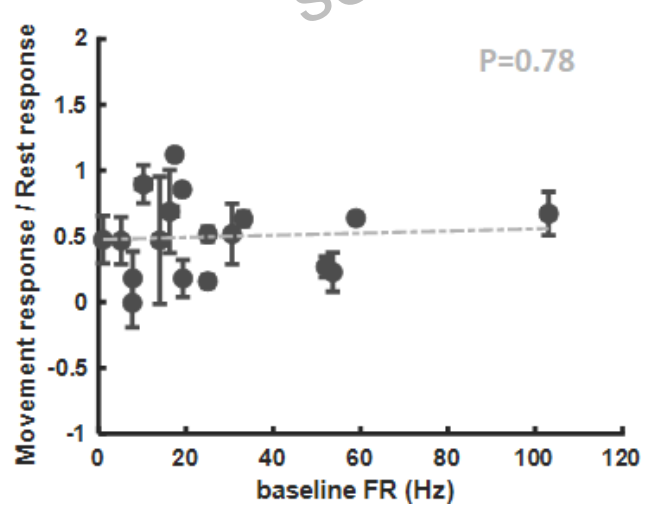

B

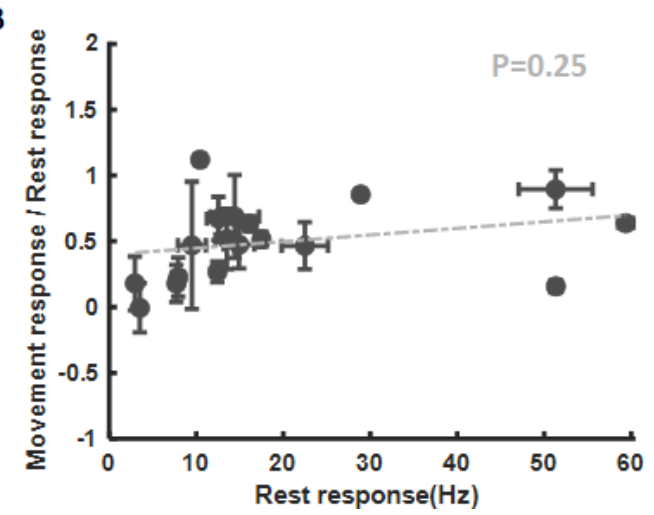

Supplementary Figure 2. Saturation effects analysis. A| Effect of baseline firing rates on the modulation effect. Slope of fitted (dashed) line is not significantly different from 0 . B| Effect of stimulus-evoked response at rest on the modulation effect. The slope of fitted (dashed) line is not significantly different from 0 . 


\section{REFERENCES}

Aimonetti, J.-M., Hospod, V., Roll, J.-P., \& Ribot-Ciscar, E. (2007). Cutaneous afferents provide a neuronal population vector that encodes the orientation of human ankle movements. The Journal of Physiology, 580(2), 649-658. https://doi.org/10.1113/jphysiol.2006.123075

Aimonetti, J.-M., Roll, J.-P., Hospod, V., \& Ribot-Ciscar, E. (2012). Ankle joint movements are encoded by both cutaneous and muscle afferents in humans. Experimental Brain Research, 221(2), 167-176. https://doi.org/10.1007/s00221-012-3160-2

Angel, R. W., \& Malenka, R. C. (1982). Velocity-dependent suppression of cutaneous sensitivity during movement. Experimental Neurology, 77(2), 266-274. https://doi.org/10.1016/0014-4886(82)90244-8

Biedenbach, M. A. (1972). Cell density and regional distribution of cell types in the cuneate nucleus of the Rhesus monkey. Brain Research, 45(1), 1-14. https://doi.org/10.1016/0006-8993(72)90212-0

Chakrabarti, S., \& Schwarz, C. (2018). Cortical modulation of sensory flow during active touch in the rat whisker system. Nature Communications, 9(1), 3907. https://doi.org/10.1038/s41467-018-06200-6

Chapman, C. E., Bushnell, M. C., Miron, D., Duncan, G. H., \& Lund, J. P. (1987). Sensory perception during movement in man. Experimental Brain Research, 68(3), 516-524. https://doi.org/10.1007/BF00249795

Chapman, C. E., Jiang, W., \& Lamarre, Y. (1988). Modulation of lemniscal input during conditioned arm movements in the monkey. Experimental Brain Research, 72(2). https://doi.org/10.1007/BF00250254

Cohen, L. G., \& Starr, A. (1985). Vibration and muscle contraction affect somatosensory evoked potentials. Neurology, 35(5), 691-691. https://doi.org/10.1212/WNL.35.5.691

Colino, F. L., Buckingham, G., Cheng, D. T., van Donkelaar, P., \& Binsted, G. (2014). Tactile gating in a reaching and grasping task. Physiological Reports, 2(3), e00267. https://doi.org/10.1002/phy2.267

Confais, J., Kim, G., Tomatsu, S., Takei, T., \& Seki, K. (2017). Nerve-Specific Input Modulation to Spinal Neurons during a Motor Task in the Monkey. The Journal of Neuroscience, 37(10), 2612-2626.

https://doi.org/10.1523/JNEUROSCI.2561-16.2017

Conner, J. M., Bohannon, A., Igarashi, M., Taniguchi, J., Baltar, N., \& Azim, E. (2021). Modulation of tactile feedback for the execution of dexterous movement [Preprint]. Neuroscience. https://doi.org/10.1101/2021.03.04.433649

Costanzo, R. M., \& Gardner, E. P. (1981). Multiple-joint neurons in somatosensory cortex of awake monkeys. Brain Research, 214(2), 321-333. https://doi.org/10.1016/0006-8993(81)91197-5

Darian-Smith, C., \& Ciferri, M. (2006). Cuneate nucleus reorganization following cervical dorsal rhizotomy in the macaque monkey: Its role in the recovery of manual dexterity. The Journal of Comparative Neurology, 498(4), 552565. https://doi.org/10.1002/cne.21088

Edin, B. B., \& Abbs, J. H. (1991). Finger movement responses of cutaneous mechanoreceptors in the dorsal skin of the human hand. Journal of Neurophysiology, 65(3), 657-670. https://doi.org/10.1152/jn.1991.65.3.657

Ghez, C., \& Pisa, M. (1972). Inhibition of afferent transmission in cuneate nucleus during voluntary movement in the cat. In Brain Research (Vol. 40, Issue 1, pp. 145-151). https://doi.org/10.1016/0006-8993(72)90120-5

Goodman, J. M., \& Bensmaia, S. J. (2018). The Neural Basis of Haptic Perception. In Stevens' Handbook of Experimental Psychology and Cognitive Neuroscience (pp. 1-39). American Cancer Society.

https://doi.org/10.1002/9781119170174.epcn205 
Johansson, R. S. (1978). Tactile sensibility in the human hand: Receptive field characteristics of mechanoreceptive units in the glabrous skin area. The Journal of Physiology, 281(1), 101-125.

https://doi.org/10.1113/jphysiol.1978.sp012411

Johansson, R. S., \& Flanagan, J. R. (2009). Coding and use of tactile signals from the fingertips in object manipulation tasks. Nature Reviews. Neuroscience, 10(5), 345-359. https://doi.org/10.1038/nrn2621

Juravle, G., Binsted, G., \& Spence, C. (2017). Tactile suppression in goal-directed movement. Psychonomic Bulletin \& Review, 24(4), 1060-1076. https://doi.org/10.3758/s13423-016-1203-6

Juravle, G., McGlone, F., \& Spence, C. (2013). Context-dependent changes in tactile perception during movement execution. Frontiers in Psychology, 4. https://doi.org/10.3389/fpsyg.2013.00913

Kurz, M. J., Wiesman, A. I., Coolidge, N. M., \& Wilson, T. W. (2018). Haptic exploration attenuates and alters somatosensory cortical oscillations. Journal of Physiology, 596(20), 5051-5061. https://doi.org/10.1113/JP276263

Loutit, A. J., Vickery, R. M., \& Potas, J. R. (2020). Functional Organisation and Connectivity of the Dorsal Column Nuclei Complex Reveals a Sensorimotor Integration and Distribution Hub [Preprint]. BIOLOGY.

https://doi.org/10.20944/preprints201911.0084.v3

Mathis, A., Mamidanna, P., Cury, K. M., Abe, T., Murthy, V. N., Mathis, M. W., \& Bethge, M. (2018). DeepLabCut: Markerless pose estimation of user-defined body parts with deep learning. Nature Neuroscience, 21(9), 12811289. https://doi.org/10.1038/\$41593-018-0209-y

Post, L. J., Zompa, I. C., \& Chapman, C. E. (1994). Perception of vibrotactile stimuli during motor activity in human subjects. Experimental Brain Research,100(1). https://doi.org/10.1007/BF00227283

Qi, H.-X., \& Kaas, J. H. (2006), Organization of primary afferent projections to the gracile nucleus of the dorsal column system of primates. The Journal of Comparative Neurology, 499(2), 183-217.

https://doi.org/10.1002/cne.21061

Rushton, D. N., Roghwell, J. C., \& Craggs, M. D. (1981). GATING OF SOMATOSENSORY EVOKED POTENTIALS DURING DIFFERENT KINDS OF MOVEMENT IN MAN. Brain, 104(3), 465-491. https://doi.org/10.1093/brain/104.3.465

Sakamoto, M., Nakajima, T., Wasaka, T., Kida, T., Nakata, H., Endoh, T., Nishihira, Y., \& Komiyama, T. (2004). Loadand cadence-dependent modulation of somatosensory evoked potentials and Soleus $\mathrm{H}$-reflexes during active leg pedaling in humans. Brain Research, 1029(2), 272-285. https://doi.org/10.1016/j.brainres.2004.09.054

Schmidt, R. F., Schady, \& Torebjork. (1990). Gating of tactile input from the hand. 6.

Seki, K., \& Fetz, E. E. (2012). Gating of Sensory Input at Spinal and Cortical Levels during Preparation and Execution of Voluntary Movement. Journal of Neuroscience, 32(3), 890-902. https://doi.org/10.1523/JNEUROSCI.4958-

11.2012

Staines, W. R., Brooke, J. D., \& Mcllroy, W. E. (2000). Task-relevant selective modulation of somatosensory afferent paths from the lower limb: NeuroReport, 11(8), 1713-1719. https://doi.org/10.1097/00001756-200006050-00024

Staines, W. R., Graham, S. J., Black, S. E., \& Mcllroy, W. E. (2002). Task-Relevant Modulation of Contralateral and Ipsilateral Primary Somatosensory Cortex and the Role of a Prefrontal-Cortical Sensory Gating System.

Neurolmage, 15(1), 190-199. https://doi.org/10.1006/nimg.2001.0953

Suresh, A. K., Greenspon, C. M., He, Q., Rosenow, J. M., Miller, L. E., \& Bensmaia, S. J. (2021). Sensory computations in the cuneate nucleus of macaques [Preprint]. Neuroscience. https://doi.org/10.1101/2021.07.28.454185 
bioRxiv preprint dol: https://doi.org/10.1101/2021.11.15.468735; this version posted December 3, 2021. The copyright holder for this preprint (which was not certified by peer review) is the author/funder, who has granted bioRxiv a license to display the preprint in perpetuity. It is made available under aCC-BY-NC-ND 4.0 International license.

Suresh, A. K., Winberry, J. E., Versteeg, C., Chowdhury, R., Tomlinson, T., Rosenow, J. M., Miller, L. E., \& Bensmaia, S. J. (2017). Methodological considerations for a chronic neural interface with the cuneate nucleus of macaques. Journal of Neurophysiology, 118(6), 3271-3281. https://doi.org/10.1152/jn.00436.2017

Versteeg, C., Rosenow, J. M., Bensmaia, S. J., \& Miller, L. E. (2021). Encoding of limb state by single neurons in the cuneate nucleus of awake monkeys. Journal of Neurophysiology, 126(2), 693-706.

https://doi.org/10.1152/jn.00568.2020

Wasaka, T., Nakata, H., Kida, T., \& Kakigi, R. (2005). Changes in the centrifugal gating effect on somatosensory evoked potentials depending on the level of contractile force. Experimental Brain Research, 166(1), 118-125. https://doi.org/10.1007/s00221-005-2333-7

Williams, S. R., Shenasa, J., \& Chapman, C. E. (1998). Time Course and Magnitude of Movement-Related Gating of Tactile Detection in Humans. I. Importance of Stimulus Location. Journal of Neurophysiology, 79(2), 947-963. https://doi.org/10.1152/jn.1998.79.2.947

14 